\title{
Telephone based survey in adults with congenital heart disease during COVID-19 pandemic
}

\author{
Alexandra A. Frogoudaki ${ }^{1}$, Dimitrios Farmakis ${ }^{2}$, Dimitrios Tsounis $^{1}$, Sotiria Liori ${ }^{1}$, \\ Konstadinos Stamoulis ${ }^{1}$, Ignatios Ikonomidis ${ }^{1}$, Gerasimos Filippatos ${ }^{1,2}$, John Parissis ${ }^{1}$ \\ ${ }^{1}$ Second Cardiology Department, ATTIKON University Hospital, \\ National and Kapodistrian University of Athens, Greece \\ ${ }^{2}$ Cyprus University Medical School, Nicosia, Cyprus
}

The coronavirus disease 2019 (COVID-19) pandemic is a rapidly evolving situation. Patient populations at higher risk include older adults, patients with lung disease, heart disease, and diabetes. Currently, general recommendations for adults living with congenital heart disease (CHD) appear no different from recommendations for the general population. The high-risk $\mathrm{CHD}$ population included patients with single ventricles or those palliated with Fontan circulation, chronic cyanosis, heart failure (HF) or cardiomyopathy, pulmonary hypertension, and significant co-existing conditions, as well as heart transplant recipients and patients with reduced immunity. This classification though, is based on expert opinions [1]. Both healthcare professionals and the CHD population have been affected by the pandemic. The lack of data and difficulty in the assessment of healthcare services are main reasons for patient anxiety.

The aim herein, was to record the impact of COVID-19 pandemic on adult CHD (ACHD) patients with and without HF, their adaptation in the current situation, their compliance to social distancing and quarantine measures and the differences between patients with $\mathrm{HF}$ and those without.

All regular outpatient clinics had been suspended since 11 March 2020 in Greece. The documented tertiary hospital ACHD clinic had already established a telephone-based follow-up system for all patients. In this context, 336 consecutive ACHD patients were contacted, (152 male, mean age $38.8 \pm 15.3$ years), between 30 March 2020 and 8 April 2020 to inform them about the service during the pandemic. They were asked about their health status, informed about how they could get their regular prescriptions without a hospital visit, explained the healthcare services which were available in case of an emergency and the availability of medical advice. They were also asked for consent to answering a questionnaire addressing the impact of COVID-19 on their mood, daily life and compliance with quarantine measures.

Of the 336 ACHD patients contacted, 146 (43.5\%) had simple CHD, 138 (41.1\%) had moderate complex CHD, while $52(15.5 \%)$ had complex CHD. The diagnoses are displayed in Figure 1. Sixty-one patients (18.2\%) did not answer one or more questions.

The majority of patients $(78.2 \%)$ had not talked to a physician before the call.

Most patients $(227,82.5 \%)$ were self-isolated and were not working or were working from home. The impact of the COVID-19 pandemic on their mood, defined as long lasting emotional state, was severe in $18.9 \%$ and moderate in $51.6 \%$, while $26.5 \%$ declared that there were not impaired by the COVID-19 pandemic.

One hundred thirty-three $(39 ; 6 \%)$ patients had $\mathrm{HF}$ as defined by signs/symptoms or HF medication documented in patient records; mean age was $44.7 \pm 16.2$ years, and $66(49.6 \%)$ were male. Patients with HF were older $(\mathrm{p}=0.012)$ and had more complex CHD $(\mathrm{p}<0.001)$. Significantly more ACHD patients with HF were totally self-isolated without going out at all, compared to those without HF (52.6\% vs. $34.5 \%, \mathrm{p}=0.001)$. More non-HF

Address for correspondence: Alexandra A. Frogoudaki, MD, PhD, FESC, Second Cardiology Department, ATTIKON University Hospital, National and Kapodistrian University of Athens, 12, Vakhylidou str., 11528, Athens, Greece, tel: +30 6974413918, fax:+30 21058323, e-mail: afrogou@otenet.gr 


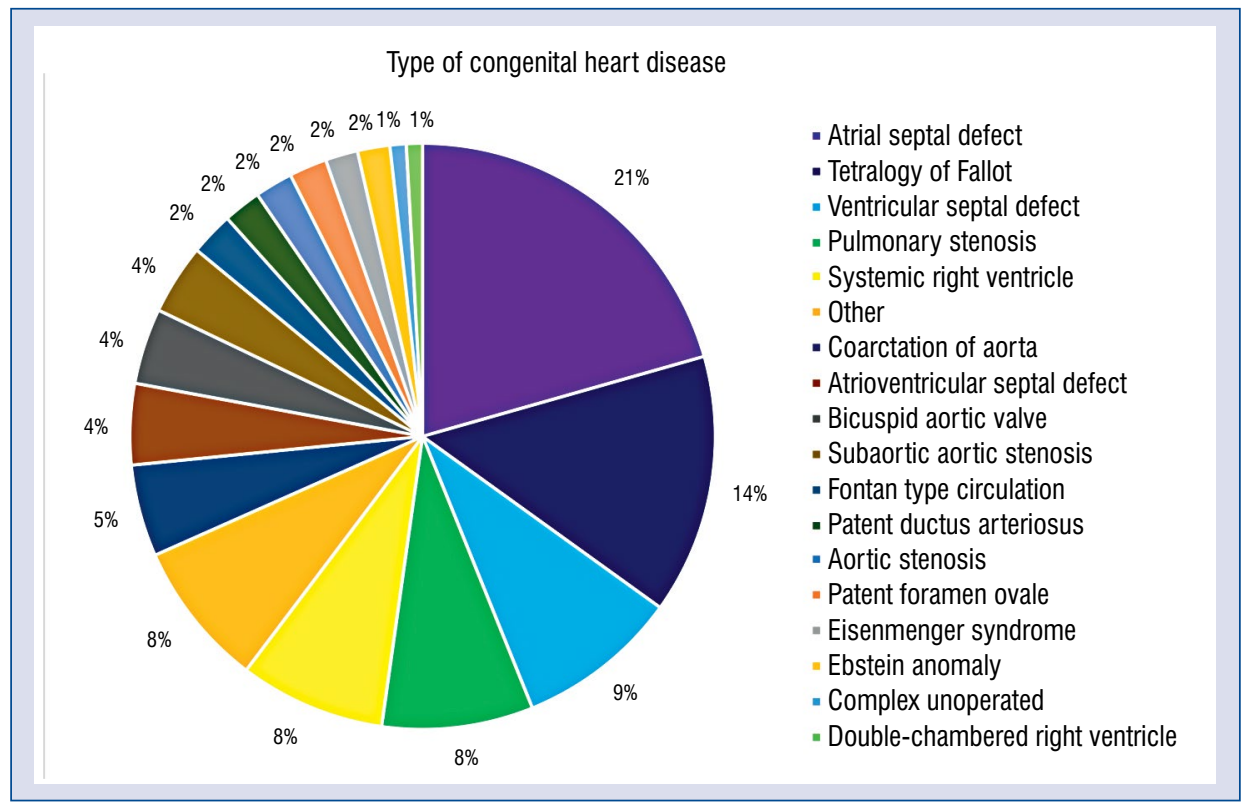

Figure 1. Congenital heart disease diagnoses.

patients stated that obligatory stay at home was the factor that impaired their mood the most $(28.6 \%$ vs. $16.5 \% \mathrm{p}=0.011$ ) compared to HF patients.

Most patients (60.8\%) were concerned for their health during the COVID-19 pandemic.

Getting infected with the COVID-19 was the main fear for ACHD patients with $\mathrm{HF}$ (50.4\% vs. $38.4 \%, \mathrm{p}=0.031$ ) while for non-HF CHD patients main concern the was unavailability of health services $(37.9 \%$ vs. $24.1 \%, p=0.008)$.

No confirmed COVID-19 cases or COVID-19 -related events occurred up to 4 weeks after being contacted.

The COVID-19 pandemic has presented a major unanticipated burden on the workforce, organizational structure, systems of care, and critical resource supply [2].

Isolation and quarantine can precipitate depression and anxiety. Many of these consequences of the pandemic will have to be addressed by psychiatrists and mental health professionals in the months to come [3].

For chronic cardiac patients i.e. ACHD, quarantine places an additional concern on how their routine follow-up is going to be impaired and how they can access health services. This can contribute to impaired mood, and potential depression. Anxiety might arise from fear of contagion and inadequate clarity about social distancing guidelines, often made worse by less reliable media sources heightening the confusion [4].
Establishing a way of communication without physical presence that enables the patients to have access to health services and reliable information on their health status and current situation enhances their security and trust, while medical issues can be safely addressed [5]. In light of all of the above, clear instructions to the high risk ACHD population were given. In this cohort, during the first few weeks of the pandemic in Greece, there were no confirmed COVID-19 infections or urgent hospital admissions.

Most patients observed restriction measures. $\mathrm{HF}$ patients self-isolated completely, which is more than ACHD patients without HF. The pandemic had an impact in the mood of the majority of patients with the effect being more prominent in $\mathrm{HF}$ patients and obligatory isolation being more disturbing for non-HF patients compared to HF patients. This may imply that asymptomatic, younger patients were more affected socially by the pandemic, while HF patients were more concerned for their health and more typically adhered to restrictions and medical treatment. Most of our ACHD patients were concerned for their health but HF patients were more afraid of getting infected with COVID-19, while non$\mathrm{HF}$ patients were concerned about the availability of health services during the pandemic.

The majority of patients observed prevention measures and followed their health status carefully. As a result, there were no COVID-19 infections or urgent admissions reported in the 
present cohort during the first few weeks of lockdown in Greece. It should be stressed that this survey took place relatively early after lockdown and does not reflect the long-term consequences of lockdown in this cohort.

In conclusion, in a large cohort of ACHD in Greece, with access to remote follow-up, most patients were aware of the prevention measures for COVID-19 infection.

No confirmed COVID-19 cases or urgent admissions for cardiac reasons were documented. Patients were largely compliant with quarantine and social distancing measures. Majority reported affected mood. CHD patients with HF were older, more afraid of getting infected with COVID-19 but were rather confident of healthcare services.

Telecommunication was useful in establishing essential contact with ACHD patients.

Conflict of interest: None declared

\section{References}

1. Tan W, Aboulhosn J. The cardiovascular burden of coronavirus disease 2019 (COVID-19) with a focus on congenital heart disease. Int J Cardiol. 2020; 309: 70-77, doi: 10.1016/j. ijcard.2020.03.063, indexed in Pubmed: 32248966.

2. Zhang SX, Wang Y, Rauch A, et al. Unprecedented disruption of lives and work: Health, distress and life satisfaction of working adults in China one month into the COVID-19 outbreak. Psychiatry Res. 2020; 288: 112958, doi: 10.1016/j.psychres.2020.112958, indexed in Pubmed: 32283450.

3. Fiorillo A, Gorwood P. The consequences of the COVID-19 pandemic on mental health and implications for clinical practice. Eur Psychiatry. 2020; 63(1): e32, doi: 10.1192/j.eurpsy.2020.35, indexed in Pubmed: 32234102.

4. Venkatesh A, Edirappuli S. Social distancing in covid-19: what are the mental health implications? BMJ. 2020; 369: m1379, doi: 10.1136/bmj.m1379, indexed in Pubmed: 32253182.

5. Hollander JE, Carr BG. Virtually Perfect? Telemedicine for COVID-19. N Engl J Med. 2020; 382(18): 1679-1681, doi: 10.1056/NEJMp2003539, indexed in Pubmed: 32160451. 